\title{
Elucidation of the thromboregulatory role of CD39/ectoapyrase in the ischemic brain
}

\author{
David J. Pinsky, ${ }^{1}$ M. Johan Broekman, ${ }^{2}$ Jacques J. Peschon, ${ }^{3}$ Kim L. Stocking, ${ }^{3}$ \\ Tomoyuki Fujita, ${ }^{1}$ Ravichandran Ramasamy, ${ }^{1}$ E. Sander Connolly, Jr., ${ }^{1}$ Judy Huang, ${ }^{1}$ \\ Szilard Kiss, ${ }^{1}$ Yuan Zhang, ${ }^{1}$ Tanvir F. Choudhri, ${ }^{1}$ Ryan A. McTaggart, ${ }^{1}$ Hui Liao, ${ }^{1}$ \\ Joan H.F. Drosopoulos, ${ }^{2}$ Virginia L. Price, ${ }^{3}$ Aaron J. Marcus, ${ }^{2}$ and Charles R. Maliszewski ${ }^{3}$
}

\begin{abstract}
${ }^{1}$ Division of Cardiology and Division of Circulatory Physiology, Department of Medicine and Department of Neurological Surgery, College of Physicians and Surgeons, Columbia University, New York, New York, USA

${ }^{2}$ Thrombosis Research Laboratory, Division of Hematology and Medical Oncology, Department of Medicine, Veterans Affairs

New York Harbor Healthcare System, and Division of Hematology and Medical Oncology, Department of Medicine and Department of Pathology, Weill Medical College of Cornell University, New York, New York, USA

${ }^{3}$ Immunex Corp., Seattle, Washington, USA
\end{abstract}

Address correspondence to: David J. Pinsky, Columbia University, College of Physicians and Surgeons,

Department of Medicine, Presbyterial Hospital 10 Stem, 630 W. 168th Street, New York, New York 10032, USA.

Phone: (212) 305-6071; Fax: (212) 305-7638; E-mail: djp5@columbia.edu.

Received for publication June 26, 2000, and accepted in revised form March 18, 2002.

\begin{abstract}
Endothelial CD39 metabolizes ADP released from activated platelets. Recombinant soluble human CD39 (solCD39) potently inhibited ex vivo platelet aggregation in response to ADP and reduced cerebral infarct volumes in mice following transient middle cerebral artery occlusion, even when given 3 hours after stroke. Postischemic platelet and fibrin deposition were decreased and perfusion increased without increasing intracerebral hemorrhage. In contrast, aspirin did not increase postischemic blood flow or reduce infarction volume, but did increase intracerebral hemorrhage. Mice lacking the enzymatically active extracellular portion of the CD39 molecule were generated by replacement of exons 4-6 (apyrase-conserved regions 2-4) with a PGKneo cassette. Although CD39 mRNA $3^{\prime}$ of the neomycin cassette insertion site was detected, brains from these mice lacked both apyrase activity and CD39 immunoreactivity. Although their baseline phenotype, hematological profiles, and bleeding times were normal, $c d 39^{-/-}$mice exhibited increased cerebral infarct volumes and reduced postischemic perfusion. solCD39 reconstituted these mice, restoring postischemic cerebral perfusion and rescuing them from cerebral injury. These data demonstrate that CD39 exerts a protective thromboregulatory function in stroke.
\end{abstract}

J. Clin. Invest. 109:1031-1040 (2002). DOI:10.1172/JCI200210649.

\section{Introduction}

Stroke is the third leading cause of death and the main cause of permanent morbidity in the United States, affecting over 450,000 patients annually (1). Our recent studies in a murine model of ischemic stroke demonstrated a pivotal role for platelets in progressive microvascular thrombosis distal to the primary obstruction of a major cerebrovascular tributary $(2,3)$. This progressive microvascular thrombosis is characterized by distal platelet and fibrin accumulation, resulting in postischemic hypoperfusion ("no reflow") and neuronal injury (2). While leukocyte adhesion receptors and recruited neutrophils contribute to postischemic hypoperfusion, postischemic hypoperfusion cannot be completely abrogated, because even in the absence of neutrophils, progressive microvascular thrombosis persists $(4,5)$. Two thrombolytic agents have been approved for treatment of stroke: recombinant tissuetype plasminogen activator (rtPA) and pro-urokinase. However, their therapeutic utility is limited due to risk of symptomatic and fatal intracranial hemorrhage (6).
In the United States, less than $1 \%$ of patients presenting to community hospitals with acute ischemic stroke receive rtPA (7). Inhibition of the final common pathway of platelet accumulation, via blockade of glycoprotein (GP) IIb/IIIa receptor-mediated platelet-platelet interactions, does reduce microvascular thrombosis in experimental stroke (2). However, as with thrombolytic agents, small excesses of a GPIIb/IIIa receptor blocker culminated in serious intracerebral hemorrhage. It is therefore important to identify novel strategies for inhibition of platelet function in acute stroke that will reduce intravascular thrombosis without increasing risk of intracerebral hemorrhage.

When the integrity of a blood vessel wall is compromised, platelets adhere to collagen in the subendothelium, leading to platelet activation and release of additional agonists: ADP, thromboxane $\mathrm{A}_{2}$, and serotonin. Of these, ADP is the most important platelet agonist and recruiting agent present in the microenvironment of the thrombus (8). There are three primary mechanisms by which endothelial cells 
maintain blood fluidity. These include local generation of nitric oxide, release of eicosanoids, and ectoapyrase activity. CD39, a transmembrane protein originally identified on lymphoid cells, whose extracellular portion exhibits apyrase activity (9), is a highly conserved, constitutively expressed enzyme that strongly inhibits platelet aggregation $(10,11)$. Following transfection of CD39 into COS cells, the cells acquire the ability to inhibit ADP-induced platelet aggregation, establishing CD39 as a prime thromboregulator $(10,12)$. Recently, a recombinant, soluble form of human CD39 (including a secretion leader but lacking transmembrane domains) was isolated from stably transfected $\mathrm{CHO}$ cells (11). This soluble CD39 (solCD39) preparation blocked aggregation induced by ADP and several other agonists in vitro, and circulated in mice with a half-life of approximately 2 days (11).

The present studies test the hypothesis that augmentation of endogenous CD39 would inhibit ADP-mediated autoamplification of platelet recruitment in distal microvessels and thereby reduce thrombosis following stroke. Since solCD39 does not interfere with primary GPIb-mediated platelet adhesion at the site of vessel damage, solCD39 administration should not, in theory, prevent a layer of platelets from forming at the site of injury or interfere with hemostatic mechanisms that prevent intracerebral hemorrhage. Our studies examine the thromboregulatory role of endogenous CD39 in stroke and the ability of solCD39 to inhibit microvascular thrombosis and confer cerebroprotection in stroke without inducing intracerebral hemorrhage.

\section{Methods}

Murine platelet aggregation. C57BL/6 mice (6-8 weeks old) were obtained from The Jackson Laboratory (Bar Harbor, Maine, USA). Untreated mice and mice treated with $4 \mathrm{mg} / \mathrm{kg}$ solCD39, or with $5 \mathrm{mg} / \mathrm{kg}$ aspirin, or with PBS, were anesthetized and heparinized $(10 \mathrm{U} / \mathrm{g})$ prior to blood collection via cardiac puncture; $80 \mu \mathrm{l}$ of $3.8 \%$ trisodium citrate was added to each $\mathrm{ml}$ of blood. Samples from six to eight mice were pooled, and platelet-rich plasma (PRP) was prepared by centrifugation at $900 \mathrm{~g}$ for 3 minutes at $20^{\circ} \mathrm{C}$, followed by $2 \mathrm{~min}$ utes at $100 \mathrm{~g}$ to eliminate residual erythrocytes and leukocytes. PRP contained $4 \times 10^{5}$ to $7 \times 10^{5}$ platelets per $\mu$ l. PRP $(200 \mu \mathrm{l})$ was preincubated for 3 minutes at $37^{\circ} \mathrm{C}$ with $100 \mu \mathrm{l}$ Tris-buffered saline buffer $(15 \mathrm{mM}$ Tris, $134 \mathrm{mM} \mathrm{NaCl}$, and $5 \mathrm{mM}$ glucose, $\mathrm{pH}$ 7.4) (11, 13, 14) in an aggregometer cuvette (Lumi-Aggregometer; Chrono-Log Corp., Havertown, Pennsylvania, USA). Platelet agonists ADP (Sigma Chemical Co., St. Louis, Missouri, USA), collagen (Hormon Chemie GmbH, Munchen, Germany), or sodium arachidonate (NuCheck Prep Inc., Elysian, Minnesota, USA) were added at the final concentrations indicated. Aggregation responses were recorded for 2-4 minutes and expressed as area under the curve (height times width at half height). All experiments were completed within 2 hours of blood collection.

Murine stroke model. We used an ischemia/reperfusion model in which a monofilament nylon suture was placed inside the lumen of the right carotid artery and advanced to occlude the origin of the right middle cerebral artery. After placement was confirmed by cessation of flow (measured using a laser Doppler flow probe), the filament was allowed to remain in place for 45 minutes and then withdrawn to establish reperfusion. Technical aspects of the surgical procedure were as follows: A midline neck incision was made and the right carotid artery was exposed. Middle cerebral artery occlusion was accomplished by advancing a $13-\mathrm{mm}$ blunt tipped 6-0 nylon suture via an arteriotomy in the external carotid stump. The external carotid artery was cauterized to secure hemostasis, and arterial flow was reestablished. The occluding suture was removed after 45 minutes and cautery was again locally applied to prevent bleeding at the arteriotomy site. Surgical staples were used for wound closure. At 23 hours after suture removal, unanesthetized mice were assigned a neurological score based on a fourtiered grading system (15): (I) normal spontaneous movements; (II) animal circling toward the right; (III) animal spinning to the right; and (IV) animal crouched on all fours, unresponsive to noxious stimuli. Following anesthesia, cerebral blood flow was again measured using a laser Doppler flow probe (for both ipsilateral and contralateral hemispheres), and blood flow was expressed as the ratio of ipsilateral flow to contralateral flow. Infarct volumes were analyzed by triphenyltetrazolium chloride staining of serial cerebral sections. Infarcted tissue appears white (viable tissue stains brick red). The volume of infarcted tissue was calculated based on planimetric analysis of serial cerebral sections. This model and these methods have previously been validated in studies of

Table 1

Hematological profile and coagulation parameters obtained from $c d 39^{-/-}$and control $\left(\operatorname{cd} 39^{+/+}\right)$mice

\begin{tabular}{|c|c|c|c|c|c|c|c|c|c|c|}
\hline Genotype & $\begin{array}{c}\text { Hgb } \\
(\mathrm{mg} / \mathrm{dl})\end{array}$ & $\begin{array}{c}\text { Plts } \\
\left(\text { cells } / \mu \mathrm{I} \times 10^{3}\right)\end{array}$ & $\begin{array}{l}\text { PT } \\
\text { sec }\end{array}$ & $\begin{array}{l}\text { PTT } \\
\text { sec }\end{array}$ & $\begin{array}{c}\text { WBCs } \\
\left(\text { cells } / \mu \mathrm{l} \times 10^{3}\right)\end{array}$ & $\begin{array}{l}\text { Segs } \\
\left(\text { cells }^{A}\right)\end{array}$ & $\begin{array}{l}\text { Bands } \\
\left(\text { cells }^{A}\right)\end{array}$ & $\begin{array}{l}\text { Lymphs } \\
\left(\text { cells }{ }^{\mathrm{A}}\right)\end{array}$ & $\begin{array}{l}\text { Monos } \\
\left(\text { cells }^{A}\right)\end{array}$ & $\begin{array}{c}\text { Eos } \\
\left(\text { cells }^{A}\right)\end{array}$ \\
\hline $\operatorname{cd} 39^{+/+}$ & $13.6 \pm 1.25$ & $799 \pm 98.0$ & 10.8 & 33.7 & $5.4 \pm 0.74$ & $42 \pm 2.0$ & $1.0 \pm 0.20$ & $52 \pm 2.4$ & $5.2 \pm 0.66$ & $0.8 \pm 0.37$ \\
\hline$c d 39^{-/-}$ & $13.2 \pm 0.57$ & $776 \pm 52.2$ & 11.5 & 39.8 & $3.5 \pm 0.40$ & $32.6 \pm 3.4$ & $0.4 \pm 0.24$ & $61.8 \pm 3.2$ & $5 \pm 0.45$ & $0.2 \pm 0.2$ \\
\hline$P$ & NS & NS & NS & NS & NS & NS & NS & NS & NS & NS \\
\hline
\end{tabular}

Blood samples from five mice were analyzed for each variable, and data are presented as mean \pm SEM. Hgb, hemoglobin; Plts, platelets; PT, prothrombin time; PTT, partial thromboplastin time; WBCs, white blood cells; Segs, segmented polymorphonuclear leukocytes; Lymphs, lymphocytes; Monos, monocytes; Eos, eosinophils; $P$, probability of a significant difference; NS, not significant. ACells per 100 white blood cells. 
a

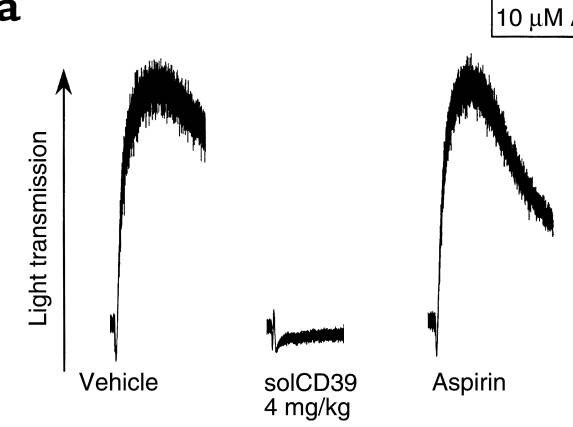

b

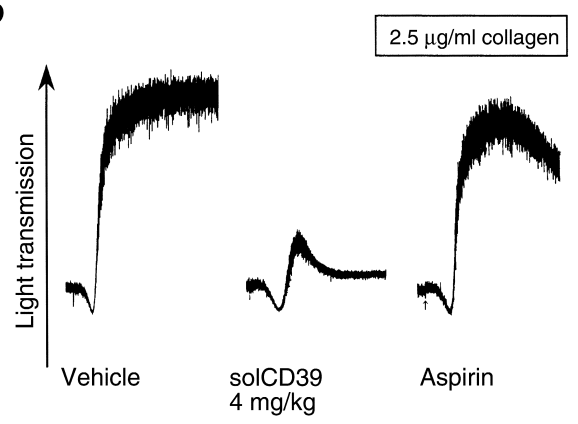

C

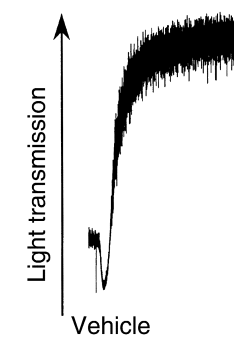

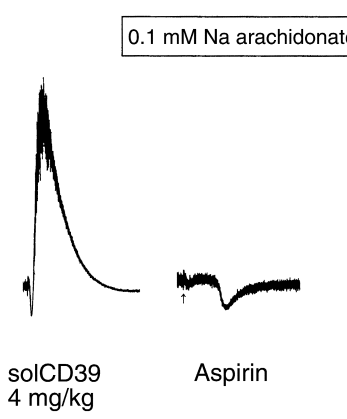

murine stroke $(2-4,15)$. Anesthetized mice were maintained at $37^{\circ} \mathrm{C} \pm 2^{\circ} \mathrm{C}$ during and for 90 minutes after surgery. Procedures for measurement of cerebral thrombosis using ${ }^{111}$ In-labeled platelets $(2,16)$, detection of intracerebral fibrin (2), and measurement of intracerebral hemorrhage $(2,17)$ have also been previously described and are described in more detail below.

Detection of intracerebral fibrin. To detect fibrin by either immunostaining or immunoblotting, 24 hours after stroke, mice were heparinized $(1,000 \mathrm{U} / \mathrm{ml}, 0.2 \mathrm{ml}$ given intravenously) about 1 minute prior to sacrifice to minimize postmortem thrombosis. After the brain was separated into right and left hemispheres, immunoblotting for fibrin was performed as originally described for

\section{Figure 2}

Effect of stroke in $c d 39^{+/+}$mice with or without solCD39 or aspirin therapy on platelet and fibrin accumulation. (a) Inhibition of platelet deposition following induction of stroke in mice pretreated with solCD39 (4 mg/ $\mathrm{kg}$ ) or aspirin (5 mg/ $\mathrm{kg}$ ). (b) Immunoblot showing fibrin accumulation following sham operation, stroke, or stroke with solCD39 $(8 \mathrm{mg} / \mathrm{kg})$ administered intravenously prior to stroke induction. The lane marked "Fibrin" represents a positive control using fibrin prepared in vitro. Ipsilat, ipsilateral (i.e., ischemic hemisphere); Contralat, nonischemic hemisphere.

\section{Figure 1}

Ex vivo aggregation of $c d 39^{+/+}$murine platelets. Following administration of saline vehicle, solCD39 $(4 \mathrm{mg} / \mathrm{kg})$, or aspirin $(5 \mathrm{mg} / \mathrm{kg})$, platelets were stimulated with $10 \mu \mathrm{M} \operatorname{ADP}(\mathbf{a}), 2.5 \mu \mathrm{g} / \mathrm{ml}$ collagen (b), or $0.1 \mathrm{mM}$ sodium arachidonate (c). SolCD39 treatment yielded aggregation curves that returned to baseline following stimulation with all three agonists. Aspirin treatment resulted in this pattern only when arachidonate was the agonist.

murine lung tissue (18). Because fibrin is extremely insoluble, brain tissue extracts were prepared by plasmin digestion, and plasmin-digested extracts were applied to a reduced $10 \%$ SDS-polyacrylamide gel $(10 \mu \mathrm{g}$ protein/lane) for subsequent electrophoresis. Following electrophoretic transfer to a nitrocellulose membrane, immunoblotting was performed using an anti-fibrin antibody (BioDesigns Inc., Saco, Maine, USA). After application of a secondary antibody (horseradish peroxidase-conjugated goat anti-rabbit $\operatorname{IgG}$ ), the enhanced chemiluminescent method (Amersham Pharmacia Biotech UK Ltd., Buckinghamshire, United Kingdom) was used to determine the location of the fibrin bands. Using NIH Image and Adobe Photoshop software, the relative intensity of the ipsilateral and contralateral bands was compared.

Measurement of intracerebral hemorrhage. Intracerebral hemorrhage was quantified using a spectrophotometric assay for hemoglobin that has been recently developed and validated for use in a murine model of stroke (17). In brief, mouse brains were homogenized, sonicated, and centrifuged, and hemoglobin in the supernatants was converted (with Drabkin's reagent) to cyanmethemoglobin, whose concentration was assessed by measuring OD at $550 \mathrm{~nm}$.

${ }^{111}$ In-platelet accumulation. Platelet accumulation was determined using ${ }^{111}$ In-labeled platelets that had been collected and prepared as previously described $(2,16$, 19-21). In brief, pooled blood was collected from control mice in $3.8 \%$ sodium citrate for anticoagulation

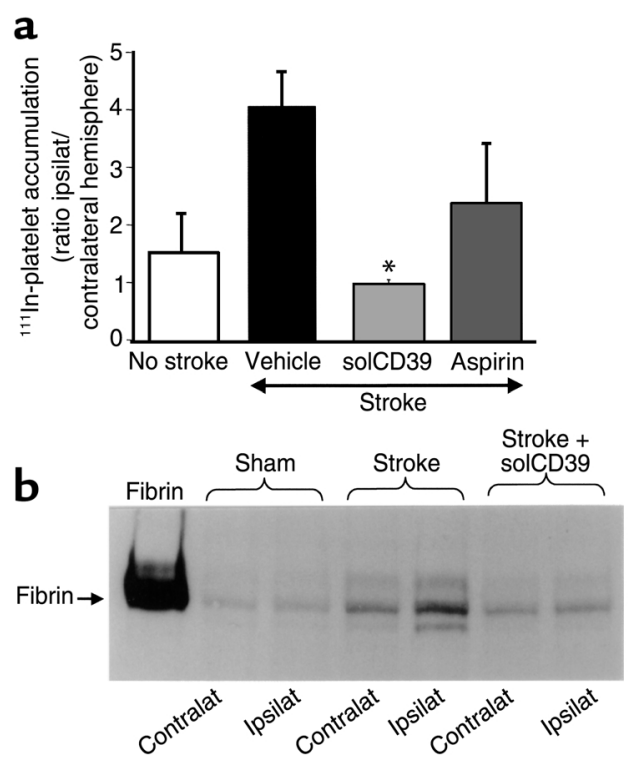


a

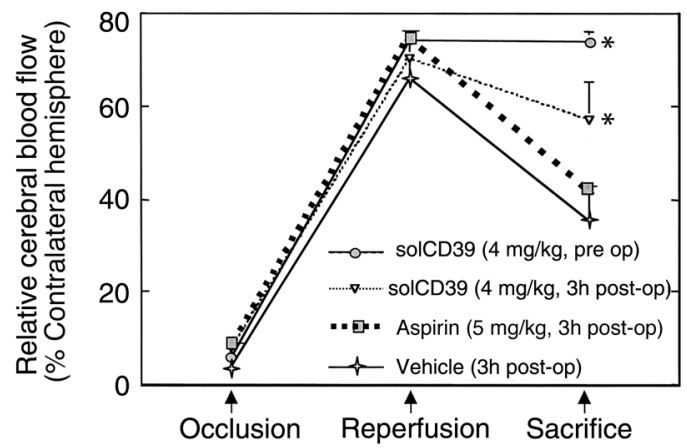

b

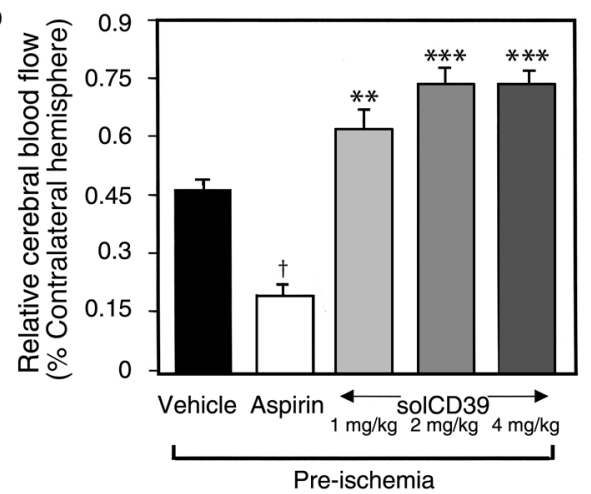

Figure 3

Comparative effects of vehicle, aspirin, and solCD39 on the various outcomes of experimental stroke in $c d 39^{+/+}$mice. (a) Relative cerebral blood flows shown at occlusion, reperfusion, and sacrifice at 23 hours after reperfusion following stroke for four treatment groups: $4 \mathrm{mg} / \mathrm{kg}$ solCD39 given preoperatively $(n=16)$ or 3 hours postoperatively $(n=9)$, aspirin given postoperatively $(n=6)$, and control (vehicle, given postoperatively; $n=10$ ). (b) Relative cerebral blood flow at 24 hours in mice treated preoperatively with vehicle $(n=24)$, aspirin $(n=27)$, or solCD39 $(n=11,11$, and 16 for the 1 $\mathrm{mg} / \mathrm{kg}, 2 \mathrm{mg} / \mathrm{kg}$, and $4 \mathrm{mg} / \mathrm{kg}$ doses, respectively). Cerebral blood flow data for vehicle or solCD39, shown in a, are repeated here for comparison. At 24 hours, the following parameters were also determined: (c) cerebral infarct volume; (d) neurological deficit score, with higher scores indicating worse deficit (15); (e) mortality; and (f) intracerebral hemorrhage. ${ }^{*} P<0.05 ; * * P<0.01 ; * * *<0.001$; ${ }^{\dagger} P<0.0001 ;{ }^{\dagger}+P=0.00002$.

(10 $\mathrm{ml}$ total). Platelets were isolated by differential centrifugation at $300 \mathrm{~g}$ for 5 minutes to obtain PRP, which was then washed three times at $2,000 \mathrm{~g}$ for 15 minutes in $10 \mathrm{ml}$ of acid/citrate/dextrose anticoagulant (ACD-A, containing $38 \mathrm{mmol} / 1$ citric acid, 75 $\mathrm{mmol} / \mathrm{l}$ sodium citrate, and $135 \mathrm{mmol} / \mathrm{l}$ glucose). The pellet was suspended in $5 \mathrm{ml}$ of ACD-A and centrifuged at $100 \mathrm{~g}$ for 5 minutes to remove contaminating red blood cells, and the supernatant was collected. ${ }^{111}$ In-oxyquinoline $(70 \mu \mathrm{l}$ of $1 \mathrm{mCi} / \mathrm{ml}$; Amersham Mediphysics, Piscataway, New Jersey, USA) was added, and the suspension was shaken gently for 30 minutes at room temperature. The radiolabeled platelets were washed three times in ACD-A and resuspended in PBS, and the platelet number was adjusted to $5 \times 10^{6} / \mathrm{ml}\left(1 \times 10^{6}\right.$ counts were given to each

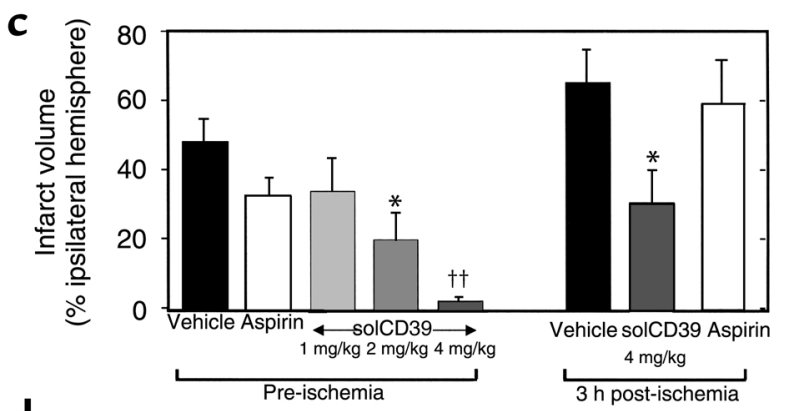

d

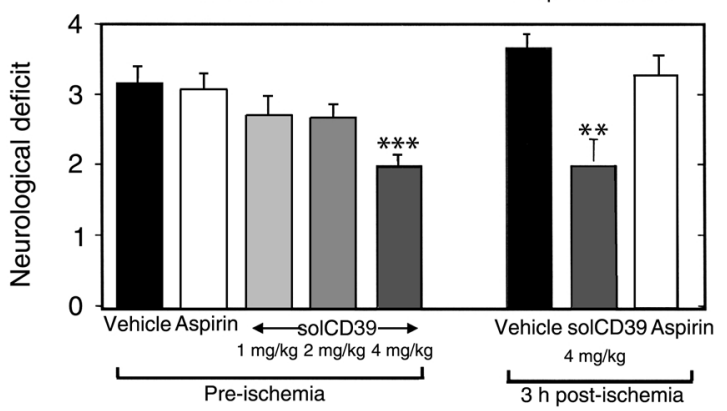

e

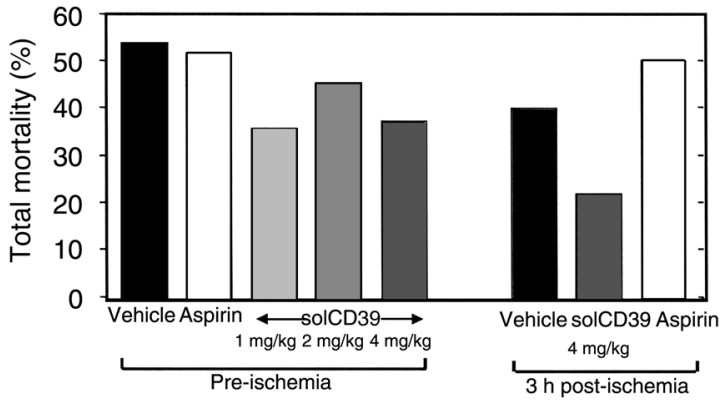

f

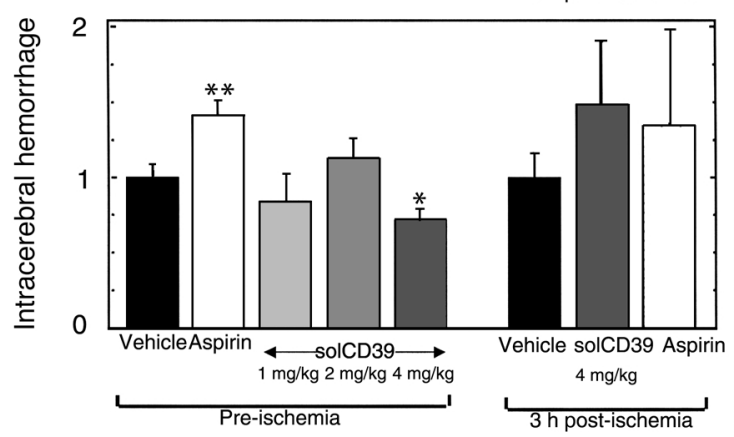

animal). Immediately prior to insertion of the occluding suture, $0.2 \mathrm{ml}$ of ${ }^{111}$ In-labeled platelet suspension was injected intravenously into the penile vein; at 23 hours after reperfusion was initiated, brain tissue was harvested and platelet accumulation was quantified as the ipsilateral/contralateral cpm ratio.

Generation of $c d 39^{--}$mice by homologous recombination. A gene-targeting vector, in which a 4.1-kb SpeI-BglII fragment containing exons 4-6 (encoding apyrase-conserved regions 2-4) (22) was replaced with a PGKneo cassette, was introduced into 129-derived embryonic stem (ES) cells, and cells were selected in G418 and ganciclovir. ES clones with a disrupted $c d 39$ allele were identified by genomic Southern blot analyses of BglIIdigested DNA and were injected into blastocysts. The resulting chimeras were crossed with $\mathrm{C} 57 \mathrm{BL} / 6$ mice to 
produce $c d 39^{+/-}$heterozygotes that were crossed to generate $c d 39^{-/-}$mice. Because strain may influence cerebral infarct volume (23) or propensity for thrombosis (24), two different control strains were used for stroke experiments in which $c d 39^{-/}$mice were studied; because of their breeding efficiency and availability, $\mathrm{C} 57 \mathrm{BL} / 6 \times 129 / \mathrm{J} \mathrm{F}_{1}$ mice served as controls for most experiments, as indicated. In a separate small cohort of animals studied to confirm these results in terms of the primary outcome measure - infarct volume $-c d 39^{-/-}$ mice bred for five generations into the C57BL/ 6 background were compared directly in blinded experiments with C57BL/ 6 controls.

Detecting expression of residual CD39 $\mathrm{mRNA}$ and protein. Probes were constructed (by PCR) for each of the intron/exon regions depicted in the schematic illustration shown in Figure $5 \mathrm{~d}$. The probe for the neomycin cassette was provided by Immunex Corp. (Seattle, Washington, USA). RNA was extracted from brains and blotted according to standard procedures (25). Immunohistochemistry, performed to detect CD39 protein, was carried out on acetone-fixed frozen sections of mouse brains. Sections were stained with a primary anti-mouse CD39 antibody (generated in rats immunized with mouse solCD39) at a 200-fold dilution for 1 hour at $37^{\circ} \mathrm{C}$, followed by application of a secondary biotin-conjugated anti-rat IgG antibody. Sections were then incubated with peroxidase-conjugated Extra-Avidin (Sigma Chemical Co.) and stained with an AEC kit (Vector Laboratories Inc., Burlingame, California, USA). Immunostained sections were then counterstained with methyl green.

Measurement of ATP and ADP levels and tissue apyrase activity. Plasma levels of ATP and ADP were determined using HPLC on neutralized perchlorate extracts of plasma samples. For the separation of nucleotides, an RP-318 Hi-Pore Reverse Phase column $(250 \times 4.6 \mathrm{~mm})$ (Bio-Rad Laboratories Inc., Hercules, California, USA) was used. A variable-wavelength UV/VIS detector (Spectra-Physics, Mountain View, California, USA) was used for detection of ATP and ADP. All compounds were quantified by integration of areas under the peak of unknowns and comparing them to those of known concentrations of standards. ADPase and ATPase enzyme activities were determined at $37^{\circ} \mathrm{C}$ in $1 \mathrm{ml}$ of a solution containing $8 \mathrm{mM} \mathrm{CaCl}_{2}, 200 \mu \mathrm{M}$ substrate (ATP for ATPase or ADP for ADPase), $50 \mathrm{mM}$ imidazole, and $50 \mathrm{mM}$ Tris, $\mathrm{pH} 7.5$ (26). The reaction was stopped by addition of $0.25 \mathrm{ml}$ of malachite green reagent, and the inorganic phosphate was estimated as described (27). One unit of ATPase (or ADPase) corresponds to release of $1 \mu \mathrm{mol}$ of inorganic phosphate $/ \mathrm{min}$ at $37^{\circ} \mathrm{C}$.

Data analysis. Values are expressed as mean \pm SEM, with the numbers of experiments performed provided in the figure legends. For experiments in which two variables were compared, the unpaired Student $t$ test was used. For experiments in which more than two variables were compared, one-way ANOVA was used, with Tukey's procedure used to test for significant differences. Contingency analysis using the Fisher exact test was performed to test for differences in mortality between various treatments. Because of the different timing used for intravenous injection (preoperatively where indicated, or after surgery for the postischemic administration studies), the data for preischemic versus postischemic agent administration were analyzed separately. This method avoids potential confounding effects of timing of hydration on outcome, or potential selection biases introduced by early differences in survival, as only those mice which survived the initial perioperative period were able to receive delayed treatment with an active or a control agent. Data were considered significantly different when $P<0.05$.

\section{Results}

Platelet aggregation: solCD39 versus aspirin. Ex vivo platelet aggregation was studied in PRP obtained from $c d 39^{+/+}$mice 1 hour after injection of saline vehicle, solCD39 (prepared as described in ref. 11), or aspirin (Figure 1). This was done to ascertain the relative potency of solCD39 as compared to aspirin, an agent that improves outcomes following transient ischemic attacks in humans (28). Platelets from control and aspirin-treated mice strongly aggregated to either ADP (Figure 1a) or collagen (Figure 1b). SolCD39 administration abrogated platelet aggregation to ADP and attenuated aggregation to collagen (Figure 1b) and arachidonate (Figure 1c). Aspirin treatment, in contrast, blocked platelet reactivity only to arachidonate (Figure 1c). Platelets from mice pretreated with solCD39 showed a brisk initial phase of aggregation to arachidonate, but before a full response occurred, the platelets rapidly disaggregated and returned to the resting state (Figure 1c). Thus arachidonate evoked an initial reaction, but the

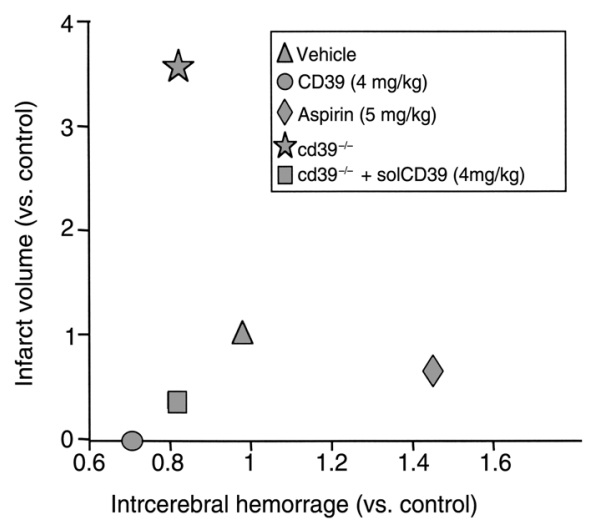

\section{Figure 4}

Covariate plot of cerebral infarct volume versus intracerebral hemorrhage as a function of genotype or treatment. Comparison of saline vehicle with aspirin $(5 \mathrm{mg} / \mathrm{kg}$ ) or solCD39 (4 mg/ $/ \mathrm{kg}$ ) given to wild-type $\left(c d 39^{+/+}\right)$mice. Data are also shown for untreated $c d 39^{-/-}$mice and cd39-/- mice given solCD39 $(4 \mathrm{mg} / \mathrm{kg})$. Data are shown for treatments given immediately prior to stroke. SEMs are shown in Figure 3, but are omitted here for clarity. 

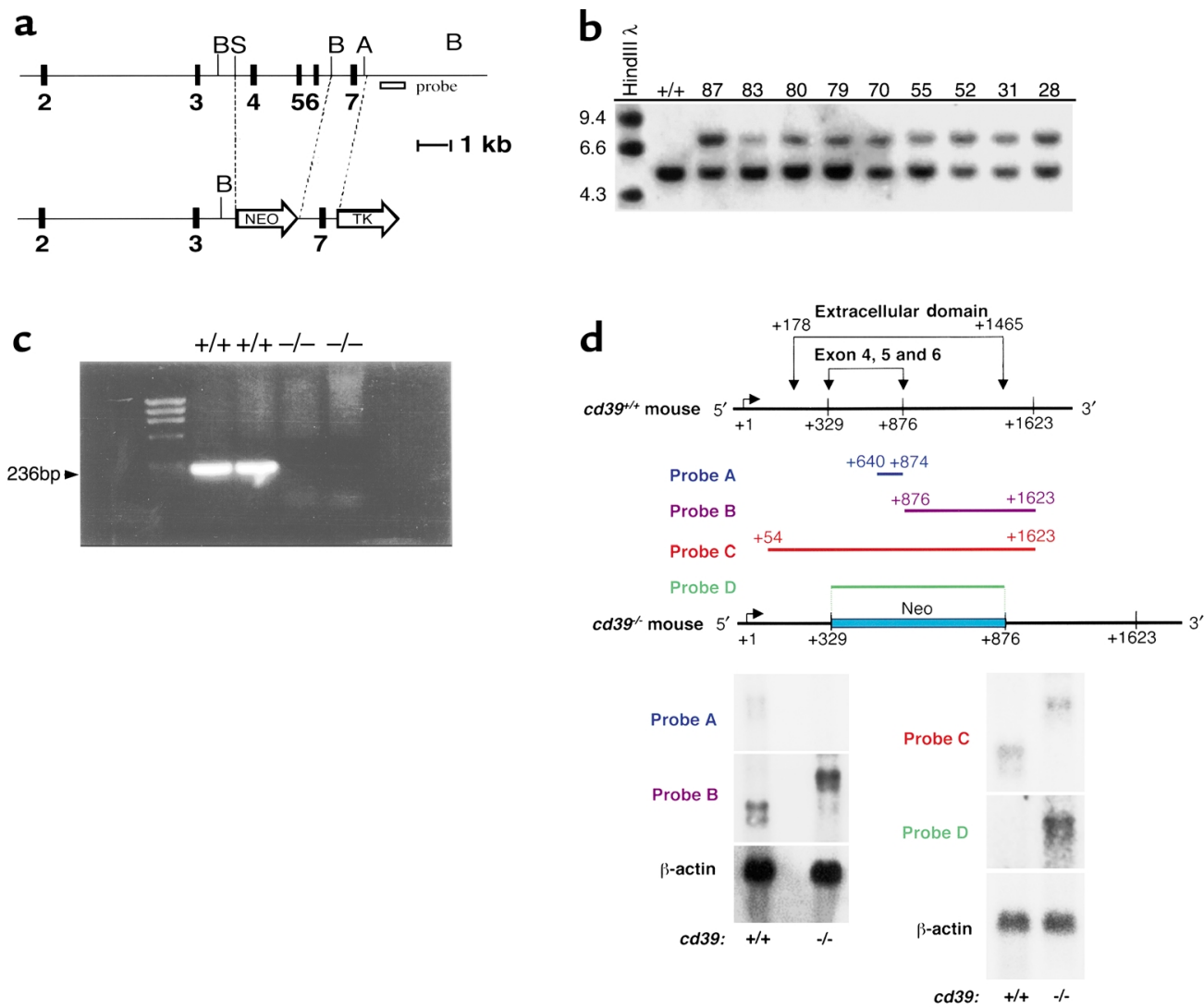

\section{Figure 5}

Generation of $c d 39^{-/-}$mice by homologous recombination. A gene targeting vector, in which a 4.1-kb Spel-Bg/ll fragment containing exons 4-6 (encoding apyrase-conserved regions 2-4) (22) was replaced with a PGKneo cassette (a), was introduced into 129-derived ES cells, and cells were selected in G418 and ganciclovir. Nine ES clones with a disrupted $c d 39$ allele, identified by genomic Southern blot analyses of Bg/lIdigested DNA (b), were injected into blastocysts and the resulting chimeras were crossed with C57BL/6 mice to produce $c d 39^{+/-}$heterozygotes. $c d 39^{-/-}$mice, generated at the expected Mendelian frequency from $c d 39^{+/-}$intercrosses, were overtly normal and did not display reproductive defects (not shown). The $c d 39^{-/-}$mice used represent random C57BL/6×129 hybrids. B, Bg/ll; S, Spel; A, Asp718. (c) Representative PCR analysis ( 25 cycles) of tail DNA, using primers within the region of the $c d 39$ gene deleted by homologous recombination (primer 1 , 5'GAACAGAGTTGGCTAAGCCTC3'; primer 2, 5'GAATGTCCTTGGCCAGTTTCTGCC3'), corresponding to a 236-bp fragment of exon 6. Each lane is from a different animal, with a genotype as indicated above the lanes. (d) Detection of expression of nonenzymatic encoding remnants of cd39 mRNA and mRNA from the neomycin cassette. The schema above the blots illustrates the regions against which probes A-D were constructed. Northern blots were performed on tissue mRNA extracts using these probes.

released ADP (required for sustaining aggregation) was metabolized by solCD39 in the PRP. This was responsible for the disaggregation observed.

Reduction in sequelae of stroke by solCD39. Experiments were performed in $c d 39^{+/+}$mice to demonstrate the therapeutic utility of intravenously injected solCD39 in stroke. SolCD39 inhibited platelet and fibrin accumulation in the ipsilateral cerebral hemisphere following induction of stroke (Figure 2, a and b). As postulated, the ability of solCD39 to reduce thrombosis was accompanied by improved postischemic cerebral perfusion (Figure 3a). In contrast, aspirin, when administered at a clinically relevant dose that inhibited the ex vivo response of platelets to arachidonate, did not improve postischemic cerebral blood flow (Figure $3 \mathrm{~b}$ ). Preoperatively administered solCD39 conferred a dosedependent diminution of cerebral infarct volumes (Figure 3c). This reduction was sustained even when solCD39 was administered 3 hours after stroke (Figure 3c). In contrast, although aspirin showed a tendency to decrease cerebral infarct volumes, the effect was not statistically significant. SolCD39 treatment (either prior to, or up to 3 hours after stroke) reduced both neurological deficit (Figure 3d) and mortality (Figure 3e).

SolCD39 and aspirin were examined with regard to development of intracerebral hemorrhage following stroke (Figure 3f). Whereas aspirin increased intracerebral hemorrhage significantly, there was no statistically significant increase in intracerebral hemorrhage at any dose of solCD39 tested (Figure 3f). At these doses, solCD39 inhibited both platelet and fibrin accumulation and promoted an increase in postischemic blood flow (Figure 2, a and b, and Figure 3a). A covariate plot of cerebral infarct volume versus intracerebral hemorrhage for each treatment indicates that aspirin is less capable of reducing infarct volume and preventing intracerebral hemorrhage than is solCD39 treatment (Figure 4). 

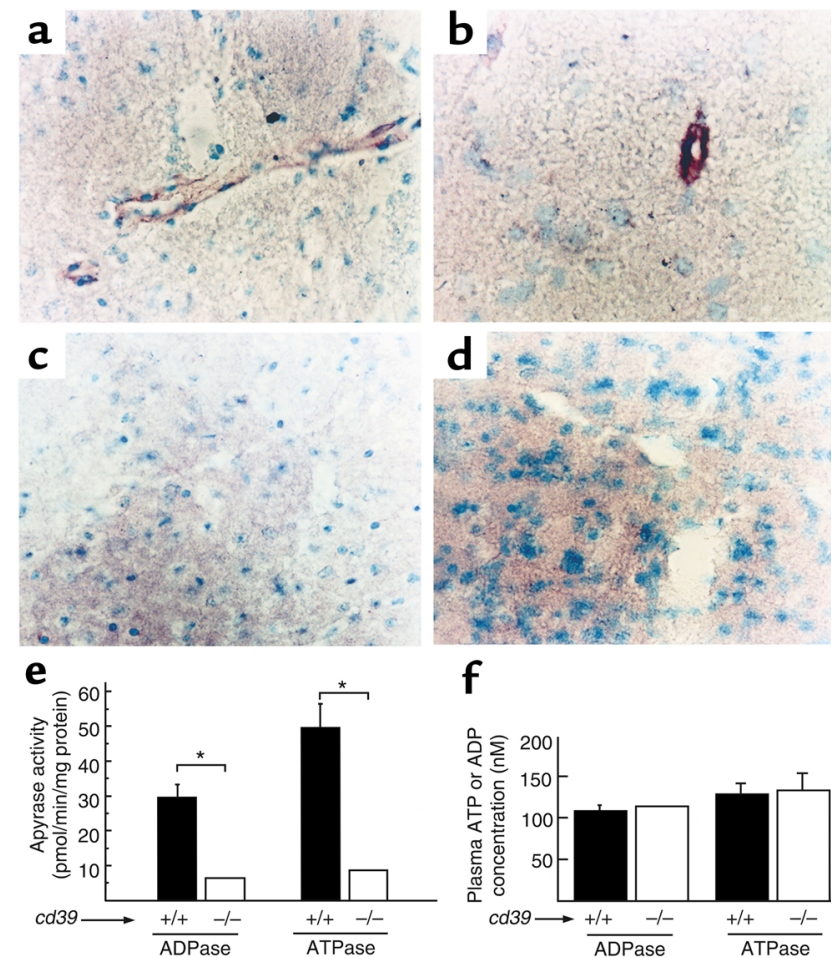

f

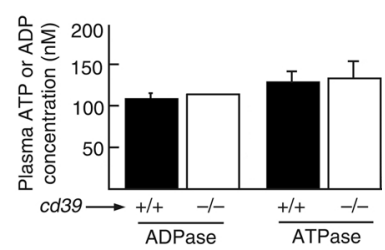

Figure 6

Expression of CD39 protein and apyrase activity. Immunostaining performed on brains of $c d 39^{+/+}$and $c d 39^{-/-}$mice. Sections from (a) cd39 $9^{+/+}$mouse, $\times 200$; (b) cd39 $9^{+/+}$mouse, $\times 400$; (c) cd39-/- mouse, $\times 200$; (d) $c d 39^{-1-}$ mouse, $\times 400$. Brain apyrase activity (e) and plasma ATP and ADP concentrations ( $f$ ) were measured using HPLC techniques after digesting samples in perchloric acid and performing neutralization with $\mathrm{KOH}$.

Reconstitution of $c d 39^{-/-}$mice with CD39. To further characterize the contributions of endogenous CD39 to hemostasis and thrombosis, $c d 39^{-/-}$mice were generated by a gene-targeting vector in which exons $4-6$, encoding apyrase-conserved regions $2-4(9,22,29,30)$, were replaced with a PGKneo cassette (Figure 5, a and b). Homozygous $c d 39^{-/-}$mice did not display an obvious phenotype in the unperturbed state. Hematological profiles were normal, including erythrocyte parameters, platelet counts, leukocyte counts and differentials, and coagulation screening tests (Table 1). To further characterize expression of $\mathrm{CD} 39$, genomic analysis demonstrated that the targeted region of the $c d 39$ gene had indeed been deleted (Figure 5c). Expression of residual cd39 mRNA in the brains of these mice was also characterized using probes designed against various regions of the $c d 39$ gene, as indicated in Figure 5d. Although residual mRNA was detected using probes for portions of cd39 located $3^{\prime}$ to the site of homologous recombination (and $3^{\prime}$ to the deletion of exons 4-6), in each case, migration of the reactive band was impeded, as expected with insertion of the neomycin cassette. This was confirmed using a probe to detect insertion of the neomycin cassette. Despite residual cryptic remnants of cd39 mRNA, immunoreactivity for CD39 protein was absent in the brains of these knockout mice. Figure 6, a and b, demonstrates immunoreactive vessels (brown staining) in longitudinal and cross section, respectively, in sections of brain taken from a $c d 39^{+/+}$mouse (positive control). In contrast, brain tissue obtained from a $c d 39^{-/-}$mouse shows no immunoreactivity, either in microvessels or elsewhere (Figure 6, c and d). Consistent with this immunohistochemical data, apyrase activity (determined by HPLC) was almost completely absent in brain tissue obtained from $c d 39^{-/-}$but not $c d 39^{+/+}$mice (Figure 6e). However, plasma levels of ADP and ATP were relatively normal in these mice (Figure 6f). Bleeding times of $c d 39^{-/-}$mice were normal, in contrast to the markedly increased bleeding time following aspirin treatment of control mice and a dose-dependent increase in bleeding time induced by solCD39 (Figure 7a). Comparison of responsiveness to $10 \mu \mathrm{M}$ ADP by platelets from $c d 39^{+/+}$ and $c d 39^{-/-}$mice indicated that platelet reactivity was somewhat reduced for the $c d 39^{-/-}$as compared with $c d 39^{+/+}$platelets (Figure 7b). However, the differences observed between CD39 null and wild-type platelets were within the overall variations obtained with different preparations of wild-type platelets.

We hypothesized that a latent prothrombotic phenotype could be identified in a clinically relevant plateletdependent stroke model (2). Indeed, $c d 39^{-/-}$mice subjected to focal cerebral ischemia did exhibit diminished blood flow following reperfusion compared with $c d 39^{+/+}$ controls (Figure 8a). When solCD39 $(4 \mathrm{mg} / \mathrm{kg})$ was

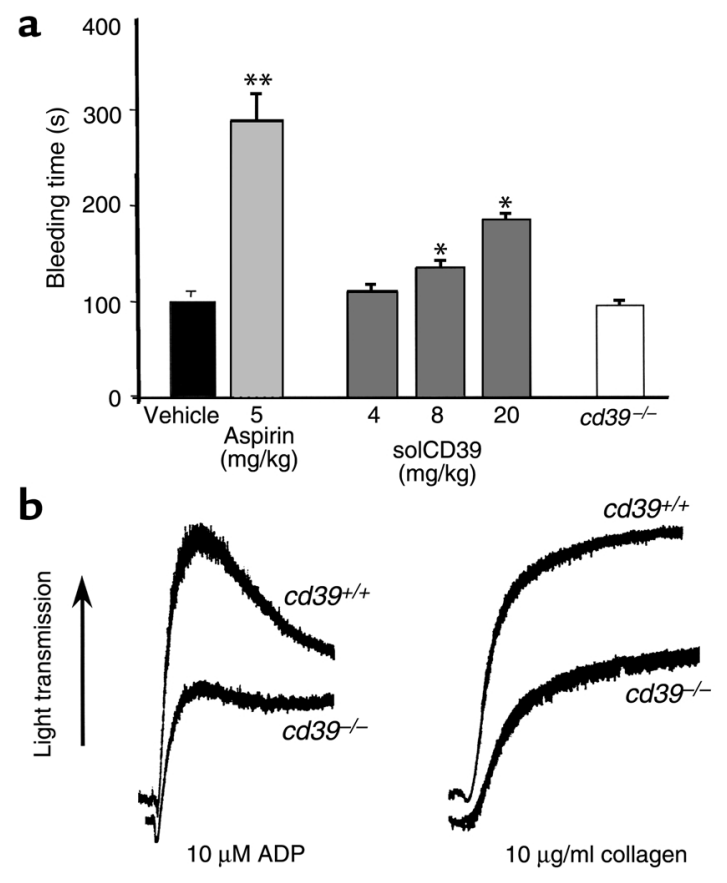

Figure 7

Role of the $c d 39$ gene, aspirin, and solCD39 in platelet function. (a) Bleeding times in control $(n=15)$, aspirin-treated $(5 \mathrm{mg} / \mathrm{kg}, n=10)$, solCD39-treated $(4,8$, and $20 \mathrm{mg} / \mathrm{kg} ; n=25)$, and CD39-/- mice $(n=10)$. The genotype of mice was $c d 39^{+/+}$unless otherwise indicated. ${ }^{*} P<0.05$. ${ }^{*} P<0.001$. (b) Comparison of responsiveness to $10 \mu \mathrm{M}$ ADP or $10 \mu \mathrm{g} / \mathrm{ml}$ collagen by platelets from $c d 39$ wild-type and null mice. Aggregometry was performed as described in the Methods section. 
a

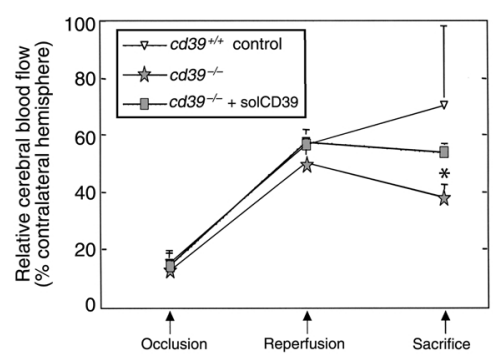

C
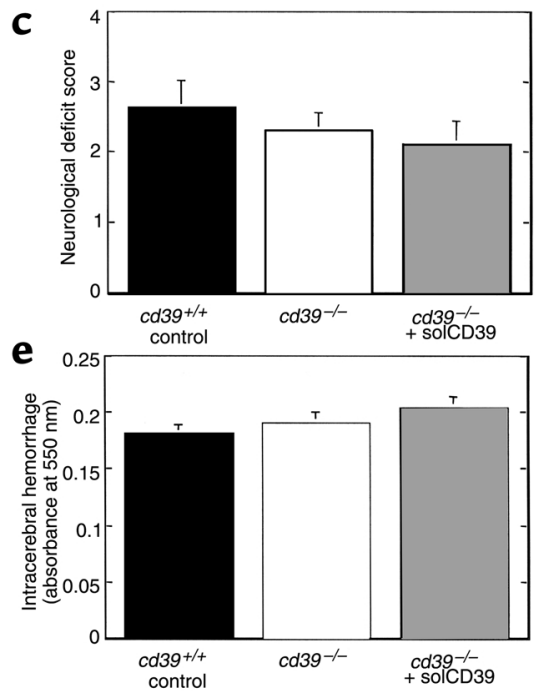

b

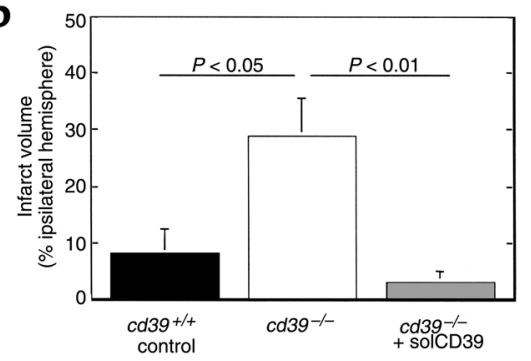

d

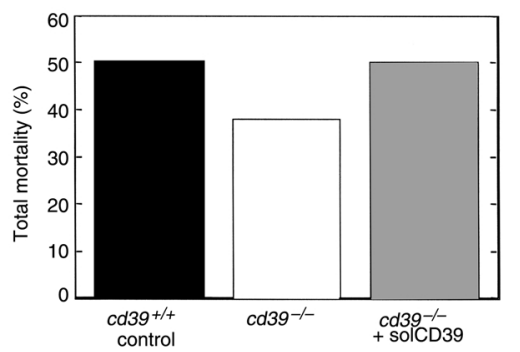

f

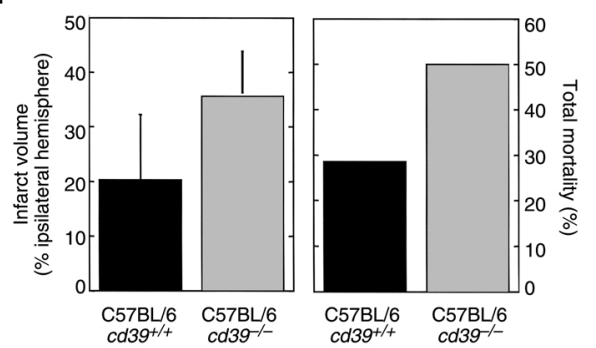

Figure 8

Comparison of stroke outcomes in $c d 39^{+/+}$control $(\mathrm{C} 57 \mathrm{BL} / 6 \mathrm{~J} \times 129 / \mathrm{J} \mathrm{F})$ mice $(n=17), c d 39^{-/-}$mice $(n=21)$, and $c d 39^{-/-}$mice reconstituted with solCD39 $(n=18)$. (a) Cerebral blood flow $\left({ }^{*} P<0.05\right.$ versus $\left.c d 39^{+/+}\right)$, (b) cerebral infarct volume, (c) neurological deficit score, $(\mathbf{d})$ mortality, and (e) intracerebral hemorrhage. Infarct volumes were compared between $c d 39^{-/-}$mice (bred for five generations into the C57BL/6 background) and $\mathrm{C} 57 \mathrm{BL} / 6$ controls in an additional cohort of animals. In this experiment, four of eight $c d 39^{-/-}$mice survived for infarct volume analysis. In the control group, five of seven mice survived for infarct volume analysis (f).

administered to the $c d 39^{-/-}$mice, these mice were "reconstituted," as shown by increased postischemic blood flow. Furthermore, $c d 39^{-/-}$mice demonstrated increased cerebral infarction volumes compared with control $c d 39^{+/+}$mice following induced stroke (Figure $8 \mathrm{~b})$. Other parameters (neurological deficit scores, overall mortality, and intracerebral hemorrhage) did not differ between groups (Figure 8, c-e). A covariate plot of infarct volume and intracerebral hemorrhage revealed that the large infarcts in $c d 39$ null mice were reduced by reconstitution with solCD39 to become similar to those in CD39-treated animals with respect to infarct volume and intracerebral hemorrhage (Figure 4). $c d 39^{-/-}$mice reconstituted with solCD39 exhibited markedly diminished infarct volumes that were similar to those in untreated $c d 39^{+/+}$controls, demonstrating a protective effect of solCD39. Although numbers of backcrossed $c d 39^{-/-}$animals (bred for five generations into the C57BL/6 background) were limited, a completely separate cohort of these backcrossed mice (with additional C57BL/ 6 controls) were subjected to stroke by an operator blinded to genotype, to serve as yet an additional control for mouse strain (Figure 8f). These data, though not achieving statistical significance, did show a trend toward exacerbation of infarct size and mortality in the $c d 39^{-/-}$mice, consonant with the other data indicating a deleterious effect on infarct volume caused by the lack of the enzymatically active portions of the $c d 39$ gene.

\section{Discussion}

Platelet and fibrin deposition downstream of an occlusive lesion contribute significantly to the postischemic hypoperfusion and tissue injury complicating stroke. Our results demonstrate for the first time in vivo protection conferred by CD39 in this platelet-dependent thrombotic disorder. SolCD39 improves cerebral blood flow and reduces cerebral infarct volume when given preoperatively. In addition, solCD39 confers significant cerebroprotection when administered 3 hours after onset of stroke. Rendering cerebroprotection at this delayed timepoint is significant because these effects occurred without an increase in mortality or intracerebral hemorrhage. The $c d 39^{-/-}$mice had a defect in thromboregulation in that they exhibited larger infarct volumes than did their $c d 39^{+/+}$controls. The $c d 39^{-1-}$ mice were reconstituted by administration of solCD39, thus fulfilling Koch's postulates (31).

$c d 39^{-/-}$mice generated by our group did not have an obvious phenotype; their baseline hematological and coagulation profiles were completely normal. Although 
probes detected residual mRNA from the $3^{\prime}$ end of $c d 39$ in brain tissue, immunoreactive CD39 protein was absent, and apyrase activity was scarcely detectable. These mice differ in several important ways from those reported by Enjyoji et al., in which the entire $c d 39$ gene was deleted (32). The Enjyoji mice paradoxically demonstrated both thrombosis and hemorrhage, with marked platelet dysfunction at baseline. This phenotype is in sharp contrast with that observed in our cd39-/- mice, in which the prothrombotic phenotype was demonstrable after induction of stroke, but baseline hematological and hemostatic parameters were normal. Platelets from our $c d 39^{-/-}$mice retain the ability to aggregate in response to ADP and collagen, and bleeding times were normal. In contrast, Enjyojii's $c d 39$ gene-deleted mice exhibited bleeding that did not stop in tail vein nicking experiments, and ex vivo reactivity of their platelets to ADP and collagen was virtually absent. Our knockout strategy focused on elimination of the enzymatically active extracellular domain (specifically, apyrase-conserved regions 2-4) of the $c d 39$ gene. In contrast, Enjyoji et al. targeted the ATG start site and a portion of the $5^{\prime} \mathrm{UTR}$.

Although the precise reasons for the marked phenotypic differences between the Enjyoji mice and the mice reported here are not known, it is possible to speculate as to potential reasons for these differences. Gene disruption may affect cell populations that may secondarily affect phenotype. For instance, mild thrombocytopenia was reported in Enjyoji et al.'s cd39 gene-deleted mice (32). Mice null for the protein P-selectin exhibit baseline leukocytosis (33). In contrast, our mice with targeted deletion of the enzymatically active region of the $c d 39$ gene exhibited both normal platelet and leukocyte counts. Moreover, we did not observe spontaneous thrombotic events, as were reported in mice overexpressing PAI-1 (34). Rather, our $c d 39^{-/-}$mice appear to exhibit a latent prothrombotic phenotype elicited by inducing a platelet-dependent thrombotic disorder (stroke). We postulate that under basal conditions, vascular homeostasis may be maintained by the endothelial thromboregulators prostacyclin and nitric oxide (35). However, a severe breach in vascular integrity leads to platelet accumulation and consequent fibrin deposition in the absence of CD39, as in the $c d 39^{-/}$mice. Functional reconstitution of our $c d 39^{-/}$mice with solCD39 normalizes the phenotype, providing a compelling case for deletion of CD39 activity as the basis for the latent prothrombotic phenotype observed in our knockout mice.

Although aspirin may be of benefit in prophylaxis of primary stroke, it does not appear to be efficacious in evolving stroke (28). Moreover, some patients obtain little benefit from aspirin (nonresponders), even though it is efficacious in others $(36,37)$. GPIIb/IIIa antagonists are potent inhibitors of platelet aggregation since they block a final step in platelet accumulation (fibrinogen bridging of surface GPIIb/IIIa receptors), thus abrogating plateletplatelet adherence. Although useful in prevention of intravascular thrombosis following percutaneous coronary intervention, these agents have not been widely studied specifically in the setting of acute stroke. One highly specific GPIIb/IIIa antagonist, GPI-562, had potent antithrombotic effects in experimental stroke, and did reduce cerebral infarction volumes, but it was associated with intracerebral hemorrhage (2). Our data show that endogenous CD39 is protective in stroke, and that administration of pharmacological doses of solCD39 is effective in inhibiting thrombosis and tissue injury in stroke. Since solCD39 inhibits all ADP-mediated platelet aggregation via metabolic deletion of ADP from the activated platelet releasate, it may be more potent than the ADP-receptor blockers currently in use.

The basis for the apparent superiority of solCD39 to aspirin may be that it induces more potent inhibition of platelet aggregation by blocking ADP-induced platelet recruitment. Thus, solCD39 is more efficient in inhibiting platelet recruitment than is aspirin via blockade of the arachidonate/thromboxane pathway. Although the hemostatic effects of agonist-induced pathways are likely to overlap with considerable redundancy in vivo, our data indicate that treatment with aspirin resulted in more bleeding in response to vein injury or stroke than did treatment with solCD39. Perhaps the initial layer of platelets that adheres to an injured vessel wall is essential for hemostasis, but in stroke, ADP-mediated recruitment of platelets into an evolving thrombus results in intravascular occlusion. SolCD39 disaggregates platelets under recruitment, but it does not have a deleterious effect on primary hemostasis.

We previously demonstrated that microvascular thrombosis is a continuing phenomenon after the onset of stroke (2). Therefore, this ongoing process can be modulated by therapeusis with solCD39, even 3 hours after stroke induction. Our data are especially pertinent in the setting of clinical observations of increased intracerebral hemorrhage when thrombolytic agents are administered beyond 3 hours after stroke onset (7). Thus, our results suggest a possible new approach to antithrombotic therapy, based upon metabolism of a major agonist for vascular occlusion: platelet-released ADP.

\section{Acknowledgments}

We thank Steven D. Gimpel, R. Guy Caspary, Louis J. Kim, and Alexander Coon for expert technical assistance. This work was supported in part by Merit Review grants from the Department of Veterans Affairs (A.J. Marcus and J.H.F. Drosopoulos), a New York Academy of Medicine Glorney-Raisbeck Medical Student Research Fellowship (R.A. McTaggart), and NIH grants HL-47073, HL-46403, and HL-07423 (A.J. Marcus, M.J. Broekman, and J.H.F. Drosopoulos); NS-02038 (E.S. Connolly, Jr.); and NS-41460, HL-59488, and HL-69448 (D.J. Pinsky). D.J. Pinsky has received unrestricted laboratory support from Immunex Corp. in the past and was an Established Investigator of the American Heart Association at the inception of these studies. 
1. Bronner, L.L., Kanter, D.S., and Manson, J.E. 1995. Primary prevention of stroke. N. Engl. J. Med. 333:1392-1400.

2. Choudhri, T.F., et al. 1998. Reduced microvascular thrombosis and improved outcome in acute murine stroke by inhibiting GP IIb/IIIa receptor-mediated platelet aggregation. J. Clin. Invest. 102:1301-1310.

3. Huang, J., et al. 1999. Neuronal protection in stroke by an sLe ${ }^{\mathrm{x}}$-glycosylated complement inhibitory protein. Science. 285:595-599.

4. Connolly, E.S., Jr., et al. 1996. Cerebral protection in homozygous null ICAM-1 mice after middle cerebral artery occlusion. Role of neutrophil adhesion in the pathogenesis of stroke. J. Clin. Invest. 97:209-216.

5. Connolly, E.S., Jr., et al. 1997. Exacerbation of cerebral injury in mice which express the P-selectin gene: identification of P-selectin blockade as a new target for the treatment of stroke. Circ. Res. 81:304-310.

6. Wardlaw, J.M., Warlow, C.P., and Counsell, C. 1997. Systematic review of evidence on thrombolytic therapy for acute ischaemic stroke. Lancet. 350:607-614.

7. Chiu, D., et al. 1998. Intravenous tissue plasminogen activator for acute ischemic stroke: feasibility, safety, and efficacy in the first year of clinical practice. Stroke. 29:18-22.

8. Marcus, A.J., and Safier, L.B. 1993. Thromboregulation: multicellular modulation of platelet reactivity in homeostasis and thrombosis. FASEB J. 7:516-522.

9. Wang, T.F., and Guidotti, G. 1996. CD39 is an ecto- $\left(\mathrm{Ca}^{2+}, \mathrm{Mg}^{2+}\right)$-apyrase. J. Biol. Chem. 271:9898-9901.

10. Marcus, A.J., et al. 1997. The endothelial cell ecto-ADPase responsible for inhibition of platelet function is CD39. J. Clin. Invest. 99:1351-1360.

11. Gayle, R., III, et al. 1998. Inhibition of platelet function by recombinant soluble ecto-ADPase/CD39. J. Clin. Invest. 101:1851-1859.

12. Kaczmarek, E., et al. 1996. Identification and characterization of CD39 vascular ATP diphosphohydrolase. J. Biol. Chem. 271:33116-33122.

13. Broekman, M.J., Eiroa, A.M., and Marcus, A.J. 1991. Inhibition of human platelet reactivity by endothelium-derived relaxing factor from human umbilical vein endothelial cells in suspension. Blockade of aggregation and secretion by an aspirin-insensitive mechanism. Blood. 78:1033-1040.

14. Marcus, A.J., et al. 1991. Inhibition of platelet function by an aspirininsensitive endothelial cell ADPase. Thromboregulation by endothelia cells. J. Clin. Invest. 88:1690-1696.

15. Connolly, E.S., Jr., Winfree, C.J., Stern, D.M., Solomon, R.A., and Pinsky, D.J. 1996. Procedural and strain-related variables significantly affect outcome in a murine model of focal cerebral ischemia. Neurosurgery. 38:523-532.

16. Naka, Y., et al. 1995. Enhanced preservation of orthotopically transplanted rat lungs by nitroglycerin but not hydralazine. Requirement for graft vascular homeostasis beyond harvest vasodilation. Circ. Res. 76:900-906

17. Choudhri, T.F., Hoh, B.L., Solomon, R.A., Connolly, E.S., Jr., and Pinsky, D.J. 1997. Use of a spectrophotometric hemoglobin assay to objectively quantify intracerebral hemorrhage in mice. Stroke. 28:2296-2302.

18. Lawson, C.A., et al. 1997. Monocytes and tissue factor promote thrombosis in a murine model of oxygen deprivation. J. Clin. Invest. 99:1729-1738.

19. Mizutani, H., et al. 1990. Analyses of thrombocytopenia in idiopathic thrombocytopenic purpura-prone mice by platelet transfer experiments between (NZW x BXSB)F1 and normal mice. Blood. 75:1809-1812.
20. Naka, Y., et al. 1996. cAMP-mediated vascular protection in an orthotopic rat lung transplant model: insights into the mechanism of action of prostaglandin E1 to improve lung preservation. Circ. Res. 79:773-783.

21. Choudhri, T.F., et al. 1999. Targeted inhibition of intrinsic coagulation limits cerebral injury in stroke without increasing intracerebral hemorrhage. J. Exp. Med. 190:91-99.

22. Schoenborn, M.A., et al. 1998. Gene structure and chromosome location of mouse Cd39 coding for an ecto-apyrase. Cytogenet. Cell Genet. 81:287-289.

23. Fujii, M., Hara, H., Meng, W., Vonsattel, J.P., and Huang, Z. 1997. Strainrelated differences in susceptibility to transient forebrain ischemia in SV129 and C57black/ 6 mice. Stroke. 28:1805-1810.

24. Cui, J., et al. 2000. Spontaneous thrombosis in mice carrying the factor V Leiden mutation. Blood. 96:4222-4226.

25. Pinsky, D.J., et al. 1998. Coordinated induction of plasminogen activator inhibitor-1 (PAI-1) and inhibition of plasminogen activator gene expression by hypoxia promotes pulmonary vascular fibrin deposition. J. Clin. Invest. 102:919-928.

26. Picher, M., Sevigny, J., D’Orleans-Juste, P., and Beaudoin, A.R. 1996. Hydrolysis of P2-purinoceptor agonists by a purified ectonucleotidase from the bovine aorta, the ATP-diphosphohydrolase. Biochem. Pharmacol. 14:1453-1460.

27. Baykov, A.A., Evtushenko, O.A., and Avaeva, S.M. 1988. A malachite green procedure for orthophosphate determination and its use in alkaline phosphatase-based enzyme immunoassay. Anal. Biochem. 171:266-270.

28. Dippel, D. 1998. The results of CAPRIE, IST, and CAST. Thromb. Res. 92:S13-S16.

29. Handa, M., and Guidotti, G. 1996. Purification and cloning of a soluble ATP-diphosphohydrolase (apyrase) from potato tubers (Solanum tuberosum). Biochem. Biophys. Res. Commun. 218:916-923.

30. Maliszewski, C.R., et al. 1994. The CD39 lymphoid cell activation antigen. Molecular cloning and structural characterization. J. Immunol. 153:3574-3583.

31. Koch, R. 1891. Ueber bakteriologische Forschung. Verhandlungen des $X$ Internationalen Medizinischen Congresses Berlin. 1:35-47. (Abstr.)

32. Enjyoji, K., et al. 1999. Targeted disruption of cd39/ATP diphosphohydrolase results in disordered hemostasis and thromboregulation. Nat. Med. 5:1010-1017.

33. Mayadas, T.N., Johnson, R.C., Rayburn, H., Hynes, R.O., and Wagner, D.D. 1993. Leukocyte rolling and extravasation are severely compromised in P-selectin deficient mice. Cell. 74:541-554.

34. Erickson, L.A., et al. 1990. Development of venous occlusions in mice transgenic for the plasminogen activator inhibitor-1 gene. Nature. 346:74-76

35. Marcus, A.J. 1999. Platelets: their role in hemostasis, thrombosis, and inflammation. In Inflammation: basic principles and clinical correlates. J.I. Gallin and R. Snyderman, editors. Lippincott, Williams \& Wilkins. Philadelphia, Pennsylvania, USA. 77-95

36. Harbison, J.W. 1998. Clinical considerations in selecting antiplatelet therapy in cerebrovascular disease. Am. J. Health Syst. Pharm. 55:S17-S20.

37. Grotemeyer, K.H. 1991. Effects of acetylsalicylic acid in stroke patients. Evidence of nonresponders in a subpopulation of treated patients. Thromb. Res. 63:587-593. 